\title{
Characterization of the 'White' Appearing Clots that Cause Acute Ischemic Stroke
}

\author{
Oana Madalina Mereuta, ${ }^{a}{ }^{\mathrm{b}}$ Rosanna Rossi, ${ }^{\mathrm{a} b \mathrm{~b}}$ Andrew Douglas, ${ }^{\mathrm{a} b \mathrm{~b}}$ \\ Sara Molina Gil, ${ }^{a ’ b}$ Seán Fitzgerald, ${ }^{a ’ b}$ Abhay Pandit, ${ }^{a}$ Ray McCarthy, ${ }^{c}$ \\ Michael Gilvarry, ${ }^{\mathrm{c}}$ Eric Ceder, ${ }^{\mathrm{d}}$ Dennis Dunker, ${ }^{\mathrm{d}}$ Annika Nordanstig, \\ Petra Redfors, ${ }^{\mathrm{e}}$ Katarina Jood, ${ }^{\mathrm{e}}$ Georgios Magoufis, ${ }^{\mathrm{f}}$ Klearchos Psychogios, ${ }^{\text {f'g }}$ \\ Georgios Tsivgoulis, ${ }^{\text {g }}$ Alan O'Hare, ${ }^{\text {h }}$ Sarah Power, ${ }^{\text {h }}$ Paul Brennan, ${ }^{\text {, }}$ \\ András Nagy, ${ }^{i}$ Ágnes Vadász, ${ }^{\mathrm{i}}$ Waleed Brinjikji, ${ }^{j}$ David F Kallmes, ${ }^{j}$ \\ Istvan Szikora, ${ }^{\mathrm{i}}$ Alexandros Rentzos, ${ }^{\mathrm{d}}$ Turgut Tatlisumak, \\ John Thornton, ${ }^{\mathrm{h}}$ and Karen M. Doyle, ${ }^{\mathrm{a} b \mathrm{~b}}$
}

\begin{abstract}
Objectives: Most clots retrieved from patients with acute ischemic stroke are 'red' in color. 'White' clots represent a less common entity and their histological composition is less known. Our aim was to investigate the composition, imaging and procedural characteristics of 'white' clots retrieved by mechanical thrombectomy. Materials and methods: Seventy five 'white' thrombi were selected by visual inspection from a cohort of 760 clots collected as part of the RESTORE registry. Clots were evaluated histopathologically. Results: Quantification of Martius Scarlett Blue stain identified platelets/other as the major component in 'white' clots' (mean of $55 \%$ of clot overall composition) followed by fibrin (31\%), red blood cells $(6 \%)$ and white blood cells $(3 \%)$. 'White' clots contained significantly more platelets/other $\left(\mathrm{p}<0.001^{*}\right)$ and collagen/calcification $\left(\mathrm{p}<0.001^{*}\right)$ and less red blood cells $\left(\mathrm{p}<0.001^{*}\right)$ and white blood cells $\left(\mathrm{p}=0.018^{*}\right)$ than 'red' clots. The mean platelet and von Willebrand Factor expression was $43 \%$ and $24 \%$, respectively. Adipocytes were found in four cases. 'White' clots were significantly smaller $\left(\mathrm{p}=0.016^{*}\right)$, less hyperdense $\left(\mathrm{p}=0.005^{*}\right)$ on computed tomography angiography/non-contrast CT and were associated with a smaller extracted clot area $\left(\mathrm{p}<0.001^{*}\right)$ than 'red' clots. They primarily caused the occlusion of middle cerebral artery, were less likely to be removed by aspiration and more likely to require rescue-therapy for retrieval. Conclusions: 'White' clots represented $14 \%$ of our cohort and were platelet, von Willebrand Factor and collagen/calcification-rich. 'White' clots were smaller, less
\end{abstract}

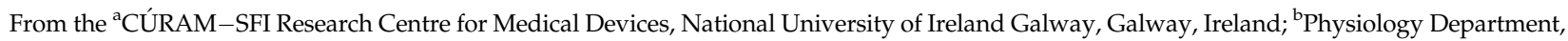
Galway Neuroscience Centre, National University of Ireland Galway, Galway, Ireland; 'Cerenovus, Galway Neuro Technology Centre, Galway, Ireland; ${ }^{\mathrm{d} D e p a r t m e n t}$ of Interventional and Diagnostic Neuroradiology, Sahlgrenska University Hospital, Institute of Clinical Sciences, Sahlgrenska Academy at University of Gothenburg, Gothenburg, Sweden; ${ }^{\mathrm{C}}$ Department of Clinical Neurosciences, Institute of Neuroscience and Physiology, Sahlgrenska Academy at University of Gothenburg and Department of Neurology, Sahlgrenska University Hospital, Gothenburg, Sweden; ${ }^{\mathrm{f}}$ Metropolitan Hospital, Stroke Unit, Piraeus, Greece; 'Second Department of Neurology, "Attikon" University Hospital, School of Medicine, National \& Kapodistrian University of Athens, Athens, Greece; ${ }^{\text {h}}$ Radiology Department, Beaumont Hospital and Royal College of Surgeons Ireland, Dublin, Ireland; 'Department of Neurointerventions, National Institute of Neurosciences, Budapest, Hungary; and 'Department of Radiology, Mayo Clinic, Rochester, Minnesota, United States.

Received June 30, 2021; revision received August 25, 2021; accepted September 14, 2021.

The study was performed within the Physiology Department, National University of Ireland Galway, Galway, Ireland.

Corresponding author at: Physiology Department, National University of Ireland Galway, Ireland. E-mail: karen.doyle@nuigalway.ie.

$1052-3057 / \$$ - see front matter

(c) 2021 The Author(s). Published by Elsevier Inc. This is an open access article under the CC BY license

(http://creativecommons.org/licenses/by/4.0/)

https://doi.org/10.1016/j.jstrokecerebrovasdis.2021.106127
} 
hyperdense, were associated with significantly more distal occlusions and were less successfully removed by aspiration alone than 'red' clots.

Key Words: Ischemic stroke-Platelets-Macroscopic appearance-Mechanical thrombectomy

(C) 2021 The Author(s). Published by Elsevier Inc. This is an open access article under the CC BY license (http:/ / creativecommons.org/licenses/by/4.0/)

\section{Introduction}

Several studies have highlighted the variations in the histopathological features of acute ischemic stroke clots (AIS) related to etiology and the impact of thrombus composition on the effectiveness of thrombolysis and mechanical thrombectomy (MT) ${ }^{1-7}$ However, only a few papers have analyzed the homogeneous/heterogeneous aspect of color ('red', 'white', mixed), size and shape of the retrieved clots; most AIS clots are mainly 'red' and histologically show a red blood cell and fibrin-dominant pattern. ${ }^{4-7}$ 'White' clots account for a small subset of thrombi and are often perplexing to the clinician regarding the etiology. A recent study has reported that AIS 'white' clots are significantly correlated with atypical etiologies. ${ }^{8}$ However, their exact composition has been less characterized.

The aim of this study was to investigate 'white' clots retrieved by MT focusing on their composition, imaging and procedural parameters.

\section{Materials and methods}

\section{Clot collection}

This study was conducted in accordance with the ethical standards of the Declaration of Helsinki and its amendments and was approved by the regional hospital ethics committees and National University of Ireland Galway research ethics committee (16-SEPT-08).

A total of 502 patients with AIS underwent MT between March 2018 and May 2019 at four centers within the RESTORE registry. The inclusion criteria were patients $>18$ years, having undergone MT for a large vessel occlusion with embolic material available for analysis.

Data regarding suspected stroke etiology, admission National Institutes of Health Stroke Scale (NIHSS) score, recombinant tissue plasminogen activator (rt-PA) treatment, approach used for MT, number of procedural passes required to remove the clot and final modified Thrombolysis in Cerebral Infarction (mTICI) score were provided by the clinical team. Occlusion location, clot length and density were assessed prior to MT using computed tomography angiography (CTA; $0.6 \mathrm{~mm}$ slice thickness) or non-contrast computed tomography (NCCT; $0.6 \mathrm{~mm}$ slice thickness). ${ }^{9}$ Stroke etiology was self-reported at each center and classified using the Trial of Org 10172 in Acute Stroke Treatment (TOAST) system: Large Artery Atherosclerosis (LAA), cardioembolic (CE), stroke of other determined etiology and cryptogenic. We defined an aspiration case when only aspiration through a catheter was performed, a stentriever case when a stentriever was used alone or in combination with an aspiration catheter and a rescue-therapy case when the approach was switched during the procedure.

\section{Clot histology and extracted clot area analysis}

Clots were immediately placed in formalin and shipped to core laboratory. Upon arrival, gross photos of each clot were taken using a Canon EOS 1300D Camera. ImageJ software ((https://imagej.nih.gov/ij/) was utilized to measure total extracted clot area (ECA) of each clot using the gross image of the clot as previously described., 10 First the scale was set and then the polygon tool was used to draw a region of interest around a fragment of the clot, and the area of that fragment was measured individually. The total extracted clot area for each case is defined as the sum of the clot area from all clot fragments within a case.

Seventy five clots retrieved from 70 patients (out of 502 cases) were classified as 'white' based on their gross appearance by two experienced pathologists within the core lab. The clots were paraffin-embedded, sectioned and stained with Martius Scarlett Blue (MSB) to identify main components ${ }^{11}$, Masson's trichrome to verify the presence of collagen and von Kossa and Alizarin Red stainings to confirm calcification and mineralization when suspected. Specimens were immunostained for platelets (rabbit monoclonal anti-CD42b, Abcam ab227669), von Willebrand Factor (monoclonal mouse anti-human vWF, Dako M0616) and fatty-acid binding protein 4 (antiFABP4 rabbit polyclonal, Abcam ab13979) for adipocytes.

Stained slides underwent whole slide scanning (Olympus VS120) at 20x magnification. Histological and immunohistochemical quantification was performed using Orbit Image Analysis software (www.orbit.bio). ${ }^{12}$

\section{Statistical analysis}

Data were analyzed using IBM SPSS-25 software. Kolmogorov-Smirnov test indicated that quantitative variables did not follow a standard normal distribution. The nonparametric Kruskal-Wallis was applied to compare basic characteristics between 'white' clots and other cases, with pairwise comparison followed by Bonferroni correction or Chi-square test for post-hoc analysis as appropriate. A level of statistical significance for all analyses was set at $\mathrm{p}<0.05$ (two-sided). Results were expressed as mean \pm SD, median [IQ1-IQ3] or number and \% of cases, as appropriate. 


\section{Results}

\section{Patient data}

Seven-hundred and sixty clots were retrieved from 502 patients. Seventy five 'white' clots retrieved from 70 patients out of 502 patients (14\%) were selected based on the macroscopic appearance. 'White' clots represented $10 \%$ of total specimens.

There was no overall significant difference in suspected etiology between 'white' and 'red' clot cases ( $\mathrm{p}=0.077$, table 1), however there was a trend. A greater percentage of 'red' clots were of LAA etiology (19.2\% 'red' vs 10\% 'white', table 1), and a greater percentage of cardioembolic $(37.1 \%$ vs $30.6 \%)$ and cryptogenic clots $(41.4 \%$ vs $35.6 \%$ ) were 'white' (table 1). There was no difference in NIHSS baseline or final TICI score between the 'white' and 'red' clot groups (table 1). Two-hundred and nine patients $(41.6 \%)$ were treated with intravenous rt-PA in addition to MT, with no significant difference between the 'white' and 'red' clot groups $(38.6 \%$ vs $42.1 \%$ treated with rt-PA respectively, table 1). Although not significant $(\mathrm{p}=0.051$, table 1$)$, there was a clear trend in data of number of passes required to remove 'white' and 'red' clots. A higher percentage of 'red' clots were removed in pass 1 $(44.4 \%$ vs $34.3 \%)$ and a higher percentage of 'white' clots required five or more passes (table 1 ).

\section{Platelets/other represent the main component of 'white' clots}

The quantitative analysis of MSB staining (Figure 1, Table 2) identified platelets/other as the major component in 'white' clots (mean of $55 \%$ of overall clot composition, table 2), followed by fibrin (31\%), red blood cells (RBC, 6\%) and white blood cells (WBC, 3\%). 'White' clots contained significantly more platelets/other (mean of $55 \%$ versus $20 \%$; $N=760$, $\mathrm{H} 1=107.91, \mathrm{p}<0.001^{*}$ ) and less red blood cells (mean of $6 \%$ versus $46 \%$; $\mathrm{N}=760, \mathrm{H} 1=181.18, \mathrm{p}<0.001^{*}$ ) compared to the 'red' clots (685 clots retrieved from 432 patients). 'White' clots had significantly less WBC than 'red' clots $(\mathrm{N}=760, \mathrm{H} 1=5.62$, $\left.\mathrm{p}=0.018^{*}\right)$. Fibrin content was similar in 'white' and 'red' clots $(\mathrm{N}=760, \mathrm{H} 1=0.59, \mathrm{p}=0.44)$.

Immunohistochemistry for specific markers showed that platelets (CD42b) represented $43 \%$ of 'white' clot composition while the mean vWF expression was $24 \%$ (Figures 2B, 2C; Table 2). Platelet-rich regions identified by MSB stain were also CD42b and vWF-rich regions (Figure 2A).

\section{Calcification, collagen and adipocytes are present in 'white' clots}

'White' clots contained significantly more collagen and calcification than 'red' clots $(6 \%$ versus $0.09 \%, \mathrm{H} 1=61.9$, $\mathrm{p}<0.001^{*}$ ) suggesting a stiffer thrombus material. As shown in a representative case, calcification suspected on gross examination (Figure 3A) was confirmed by von
Kossa and Alizarin Red (Figure 3C). The presence of collagen was confirmed by Masson's trichrome staining in another representative case (Figure 3E).

Adipocyte-like structures were noticed in four 'white' clots. Since the lipid content is lost during processing of clots through xylene, the FABP4 antibody was used as a marker for adipocytes. Two representative cases are shown in Figure 3. FABP4 was expressed by adipocytes in the first case while in the second case, it was detected only in the nuclei in proximity to adipocytes (Figure 3D).

\section{'White' clots are associated with more distal occlusions}

There was a significant overall difference in occlusion location between 'white' and 'red' clots ( $\mathrm{p}=0.004^{*}$, Table 1$)$. The MCA was the most common occlusion site for both 'white' and 'red' clots but more 'white' clots were found in MCA (74.3\% versus $54.4 \%$ ). Post-hoc analysis showed a significantly greater percentage of 'white' clots in the MCA and significantly more 'red' clots caused occlusions of internal carotid artery compared to 'white' clots $(16.4 \%$ versus $8.6 \%$, $\left.\mathrm{X}^{2}=4.833, \mathrm{p}=0.028^{*}\right)$. 'White' clots were retrieved in M1 segment in 37 cases $(52.9 \%)$ and M2 segment in 9 cases $(12.9 \%)$. 'Red' clots were removed from M1 segment in 193 cases (44.7\%) and M2 segment in 32 cases (7.4\%).

\section{'White' clots are smaller and less hyperdense on NCCT}

We found that 'white' clots were significantly smaller on CTA/NCCT compared to the other clots (2 mm [2-3] versus $3 \mathrm{~mm}$ [2-3] ; $\mathrm{N}=406, \mathrm{H} 1=5.8, \mathrm{p}=0.016^{*}$; table 1).

'White' clots were also significantly less hyperdense compared to the other clots (57 HU [54-62.5] versus 64 HU [58.5-68]; $\left.\mathrm{N}=114, \mathrm{H} 1=7.97, \mathrm{p}=0.005^{*}\right)$. Nevertheless, presence of calcification was reflected in very high density in one case (724 HU).

\section{'White' clots have a smaller extracted clot area}

The ECA of 'white' clots was significantly smaller than the ECA of 'red' clots $\left(41 \mathrm{~mm}^{2}\right.$ [22-81] versus $23 \mathrm{~mm}^{2}$ [1257]; $\mathrm{N}=502, \mathrm{H} 1=15.8, \mathrm{p}<0.001^{*}$; table 1$)$. This finding is consistent with imaging data.

\section{'White' clots are more likely to require rescue-therapy using a stentriever for retrieval}

We found a significant difference in the overall MT approach used to extract the 'white' clots compared to 'red' clots ( $\mathrm{p}=0.006^{*}$; table 1$)$. Post hoc analysis showed that rescue-therapy was necessary to retrieve significantly more $(40 \%)$ of 'white' clots compared to $24.3 \%$ of 'red' clots. Stentrievers were used to remove a similar percentage of 'white' and 'red' clots (15.7\% versus 15.5\%) whereas significantly more 'red' clots (60.2\%) were removed by aspiration only compared to $44.3 \%$ of 'white' clots. When we considered the actual per pass device used, we observed a significant difference between 'white' 
Table 1. Clinical and intervention characteristics of study population grouped by the macroscopic appearance of clots.

\begin{tabular}{|c|c|c|c|}
\hline \multirow[t]{2}{*}{ Characteristic } & & \multicolumn{2}{|c|}{ No patients $(\%)$} \\
\hline & & 'Red' clots & 'White' clots \\
\hline & & $432(86)$ & $70(14)$ \\
\hline \multirow[t]{5}{*}{ Stroke Subtype } & Large Artery Atherosclerosis & $83(19.2)$ & $7(10)$ \\
\hline & Cardioembolic & $132(30.6)$ & $26(37.1)$ \\
\hline & Other determined etiology & $25(5.8)$ & $7(10)$ \\
\hline & Cryptogenic & $154(35.6)$ & $29(41.4)$ \\
\hline & Not available & $38(8.8)$ & $1(1.4)$ \\
\hline & \multicolumn{2}{|c|}{$\mathrm{N}=463, \mathrm{H} 1=3.12, \mathrm{p}=0.077$} \\
\hline & \multicolumn{3}{|c|}{ NIHSS Baseline } \\
\hline Mean \pm SD & & $16 \pm 6$ & $16 \pm 7$ \\
\hline \multicolumn{2}{|l|}{ Statistical analysis } & $\mathrm{N}=437, \mathrm{H} 1=$ & \\
\hline \multirow[t]{3}{*}{ IV rt-PA } & Yes & $182(42.1)$ & $27(38.6)$ \\
\hline & No & $246(57)$ & $42(60)$ \\
\hline & Not available & $4(0.9)$ & $1(1.4)$ \\
\hline \multicolumn{2}{|l|}{ Statistical analysis } & $\mathrm{N}=497, \mathrm{H} 1=$ & \\
\hline \multirow[t]{5}{*}{ No of Passes } & 1 & $192(44.4)$ & $24(34.3)$ \\
\hline & 2 & $94(21.7)$ & $18(25.7)$ \\
\hline & 3 & $56(13)$ & $4(5.7)$ \\
\hline & 4 & $34(7.9)$ & $8(11.4)$ \\
\hline & $5+$ & $56(13)$ & $16(22.9)$ \\
\hline \multicolumn{2}{|l|}{ Median [IQ1-IQ3] } & $2[1-3]$ & $2[1-4]$ \\
\hline \multicolumn{2}{|l|}{ Statistical analysis } & $\mathrm{N}=502, \mathrm{H} 1=$ & \\
\hline \multirow[t]{7}{*}{ Final mTICI score } & 0 & $5(1.2)$ & $1(1.4)$ \\
\hline & 1 & $4(0.9)$ & $4(5.7)$ \\
\hline & $2 \mathrm{a}$ & $19(4.4)$ & $4(5.7)$ \\
\hline & $2 b$ & $109(25.2)$ & $17(24.3)$ \\
\hline & $2 \mathrm{c}$ & $96(22.2)$ & $13(18.6)$ \\
\hline & 3 & $192(44.4)$ & $30(42.9)$ \\
\hline & Not available & $7(1.6)$ & $1(1.4)$ \\
\hline \multicolumn{2}{|l|}{ Statistical analysis } & \multicolumn{2}{|c|}{$\mathrm{N}=494, \mathrm{H} 1=0.73, \mathrm{p}=0.394$} \\
\hline \multirow[t]{8}{*}{ Occlusion Location } & $\mathrm{MCA}$ & $235(54.4)$ & $52(74.3)$ \\
\hline & ICA/ICA Terminus & $71(16.4)$ & $6(8.6)$ \\
\hline & Vertebrobasilar & $38(.8 .8)$ & $3(4.3)$ \\
\hline & Tandem occlusion & $54(12.5)$ & $5(7.1)$ \\
\hline & Other dual occlusion & $16(3.7)$ & $2(2.9)$ \\
\hline & Other single location & $3(0.7)$ & $1(1.4)$ \\
\hline & Multiple locations & $14(3.2)$ & $1(1.4)$ \\
\hline & Not available & $1(0.2)$ & - \\
\hline \multicolumn{2}{|l|}{ Statistical analysis } & \multicolumn{2}{|c|}{$\mathrm{N}=501, \mathrm{H} 1=8.10, \mathrm{p}=0.004 *$} \\
\hline \multicolumn{4}{|l|}{ Occlusion Length on } \\
\hline Median [IQ1-IQ3] & & $3[2-3]$ & $2[2-3]$ \\
\hline Statistical analysis & & $\mathrm{N}=406, \mathrm{H} 1=$ & \\
\hline Mean HU & & & \\
\hline Median [IQ1-IQ3] & & $64 \mathrm{HU}[58.5$ & 2.5] \\
\hline Statistical analysis & & $\mathrm{N}=114, \mathrm{H} 1=$ & \\
\hline Total ECA $\left(\mathrm{mm}^{2}\right)$ & & & \\
\hline Median [IQ1-IQ3] & & $41[22-81]$ & $23[12-57]$ \\
\hline Statistical analysis & & $\mathrm{N}=502, \mathrm{H} 1=$ & \\
\hline Overall Technique & Aspiration & $258(60.1)$ & $31(44.3)$ \\
\hline & Stentriever & $66(15.4)$ & $11(15.7)$ \\
\hline & Rescue-therapy & $105(24.5)$ & $28(40)$ \\
\hline Statistical analysis & & $\mathrm{N}=499, \mathrm{H} 1=$ & \\
\hline Per pass device & Aspiration & $494(72.1)$ & $43(57.3)$ \\
\hline & Stentriever & $190(27.7)$ & $30(40)$ \\
\hline & Not available & $1(0.2)$ & $2(2.7)$ \\
\hline Statistical analysis & & $\mathrm{N}=757, \mathrm{H} 1=$ & \\
\hline
\end{tabular}

NIHSS Score: National Institutes of Health Stroke Scale Score; IV rtPA: intravenous recombinant tissue plasminogen activator; MCA: middle cerebral artery; ICA: internal carotid artery; mTICI Score: modified Thrombolysis in Cerebral Infarction Score; NCCT: Non-contrast computed tomography; HU: Hounsfield Units; ECA: extracted clot area. 


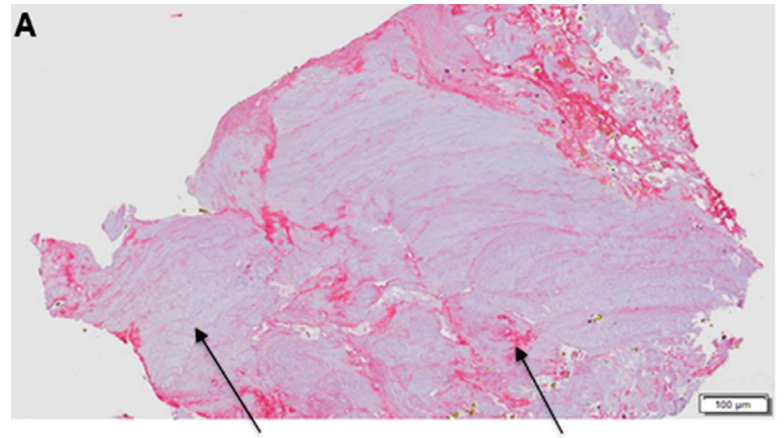

Platelets/Other (96\%) Fibrin (4\%)
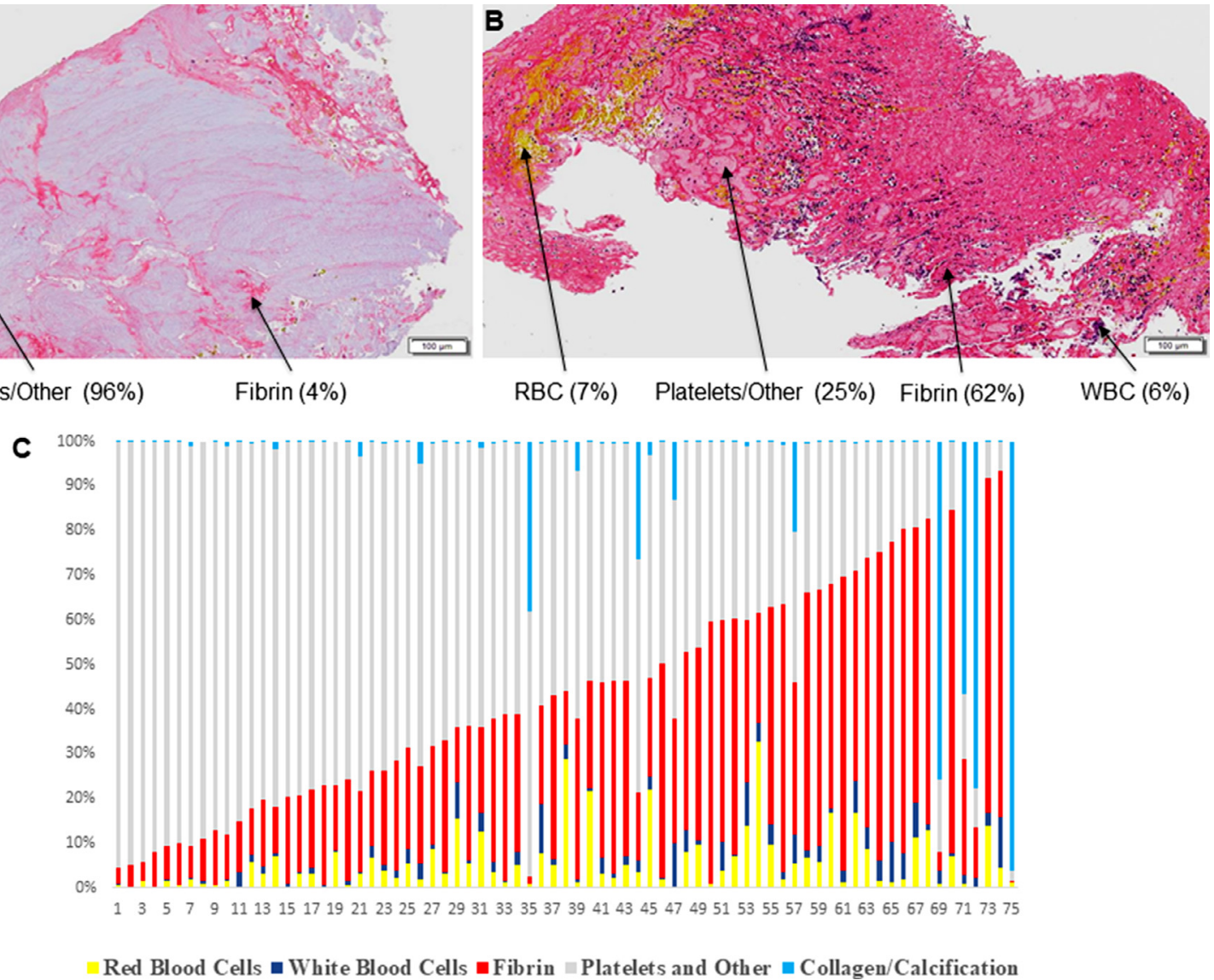

Fig. 1. 'White' clots composition. Main components identified by MSB staining in two representative platelet-rich (A) and fibrin-dominant (B) clots: red blood cells (RBC, yellow), white blood cells (WBC, blue), fibrin (red), platelets/other components (grey). Magnification: 50x (A, B). (C) Quantification of main components for each patient.

Table 2. Quantitative analysis of 'white' clots composition.

\begin{tabular}{|c|c|c|c|c|c|c|c|}
\hline & \multicolumn{4}{|c|}{ MSB stain } & \multirow[b]{2}{*}{ Collagen/Calcification } & \multicolumn{2}{|c|}{ Immunohistochemistry } \\
\hline & Platelets/other & Fibrin & $\mathrm{RBC}$ & WBC & & CD42b & vWF \\
\hline Mean \pm SD $(\%)$ & $55 \pm 25$ & $31 \pm 21$ & $6 \pm 7$ & $3 \pm 3$ & $6 \pm 18$ & $43 \pm 22$ & $24 \pm 18$ \\
\hline Range & $2-96$ & $0.2-78$ & $0.07-33$ & $0.02-11$ & $0.01-96$ & $0.04-84$ & $0.2-74$ \\
\hline
\end{tabular}

MSB: Martius Scarlett Blue; RBC: Red Blood Cells; WBC: White Blood Cells; vWF: von Willebrand factor.

clots versus 'red' clots $\left(\mathrm{p}=0.017^{*}\right.$; table 1$)$. 'White' clots were retrieved by aspiration in $57.3 \%$ of passes while aspiration catheters successfully extracted 'red' clots in $72.1 \%$ of passes. Stentrievers removed 'white' clots in $40 \%$ of passes and 'red' clots in $27.7 \%$ of passes.

\section{Discussion}

We carried out a comprehensive analysis of 75 'white' clots selected by their macroscopic aspect. We used the MSB stain which allows for a significantly better differentiation of the major components of clots than the traditional hematoxylin \& eosin stain. ${ }^{11}$ Additional stains were performed to confirm the presence of collagen (Masson's trichrome) and calcification (von Kossa). Mineralization was also demonstrated by
Alizarin Red stain suggesting that arterial calcification is not a degenerative process but is governed by similar morphogenetic signals involved in skeletal mineralization. ${ }^{13}$

'White' clots are rich in platelets, vWF and collagen/ calcification which may render them stiffer and more accessible to stentriever. Adipocytes were found occasionally. 'White' clots cause predominantly MCA occlusions and are less hyperdense on CTA/NCCT compared to 'red' clots. 'White' clots are also smaller as demonstrated by their length on CTA/NCCT and ECA measurement.

Two recent studies have shown that cardioembolic and cryptogenic clots have a smaller ECA and higher fibrin and platelets/other content compared to LAA clots which are larger and associated with a RBC-rich composition. ${ }^{10,14}$ In our cohort, the majority of 'white' 

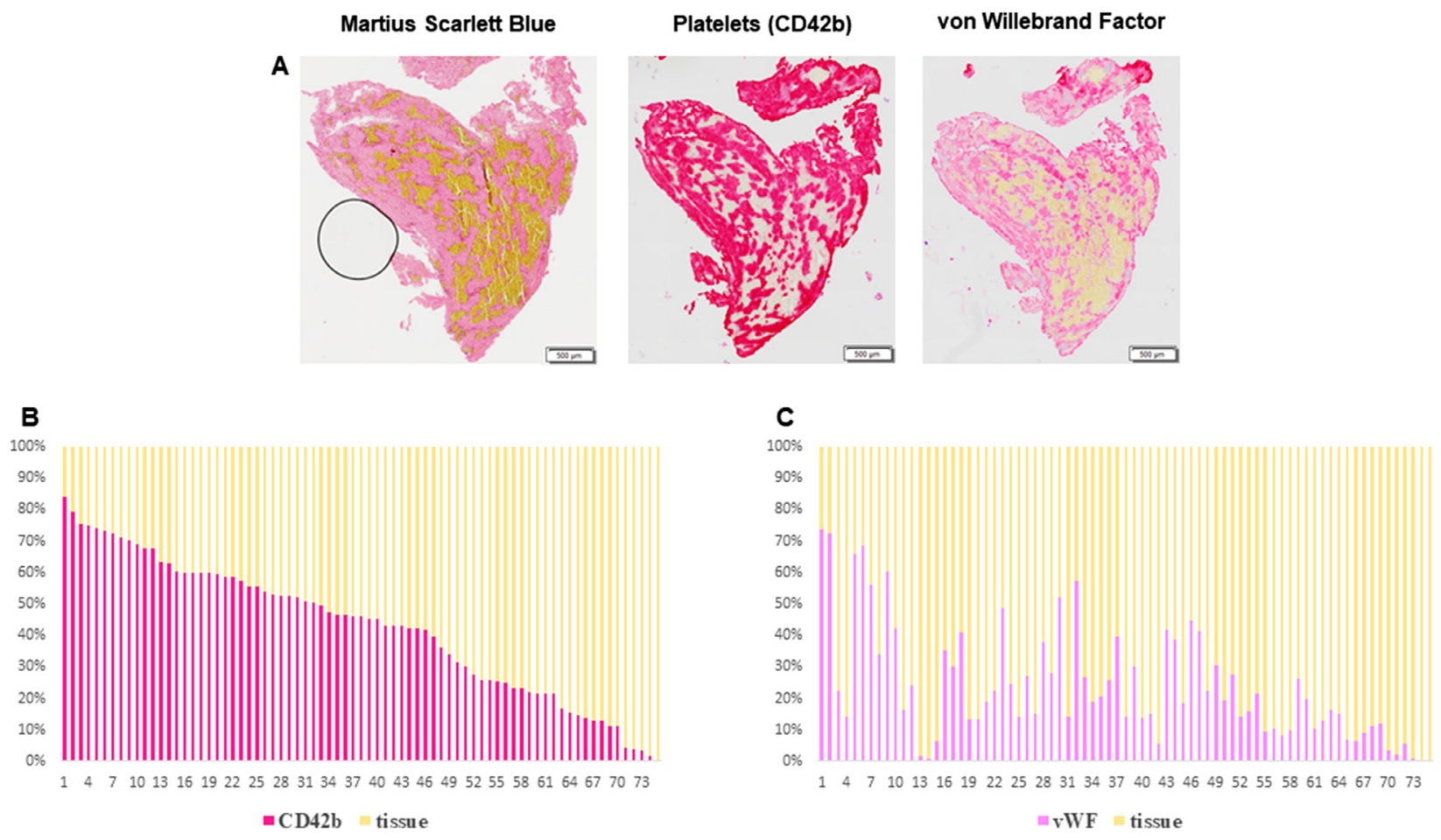

Fig. 2. Immunostaining reveals potential key components of 'white' clots. Sequential sections of a representative case stained with MSB, anti-CD42b and antivWF. Platelet-rich regions on MSB are also CD42b and $v W F-p o s i t i v e(A)$. Quantification of CD42b (B) and $v W F(C)$ expression for each clot. Magnifications: $30 x(A)$.

clots had a CE source (26 cases) or were cryptogenic $(29$ cases). In agreement with the previous studies, 'white' clots had smaller ECA and higher platelet content than other clots, characteristics that are suggestive of cardioembolic or cryptogenic etiology.

We acknowledge that there are also other components in the platelets/other-rich areas identified by MSB so we performed immunohistochemistry to distinguish between platelets and platelet-related factors such as $\mathrm{vWF}^{15}$ The high expression of CD42b and vWF in our 'white' clots highlights their critical role in 'white' clots formation. Recent studies have described important histological features that may explain the rtPA-resistance of AIS thrombi: the presence of a dense outer shell containing mainly platelets, vWF and extracellular DNA as well as the presence in the platelet-rich thrombi of dense fibrin structures lined with $\mathrm{vWF}$, extracellular DNA and filled with platelets. ${ }^{16,17}$ Given these observations, we suggest that platelet and vWF-rich composition of 'white' clots may impair thrombolysis.

Increased levels of platelets and calcium may also render 'white' clots stiffer and impact the MT outcome. ${ }^{15,18}$ However, despite the platelet and collagen/calcificationrich composition of 'white' clots compared to 'red' clots in our cohort, there was no significant difference in terms of number of passes during MT and final mTICI score. Nevertheless, aspiration alone was less effective at removing 'white' clots, presumably related to their stiff consistency. A rescue-therapy approach with a stentriever was required to remove these clots more often than 'red' clots.
We identified also the presence of adipocyte-like structures in two 'white' clots. Originally described as an adipocyte marker, FABP4 plays an important role in atherogenesis. In particular, FABP4 expression within the carotid atherosclerotic plaque is associated with its vulnerability and adverse outcome. ${ }^{19}$ FABP4 immunofluorescence confirmed the presence of adipocytes which may represent a histological marker of fat embolism or a vulnerable atherosclerotic plaque.

Imaging may hint to clot composition. ${ }^{5,6,20}$ A recent study has demonstrated that platelet-rich thrombi identified by MSB staining are isodense on NCCT. ${ }^{11}$ Boodt et al. ${ }^{6}$ showed that non-cardioembolic strokes are associated with the presence of a hyperdense artery sign, longer thrombi and an increased clot burden on CT imaging and a more proximal thrombus location. We found that 'white' thrombi are smaller and less hyperdense compared to the other clots. They were also predominantly located more distally, in the M1 or M2 segments of MCA, presumably reflecting their size.

Our findings have implications to clinical practice. Several studies have reported that the hyperdense artery sign on $\mathrm{CT}$ and the susceptibility vessel sign (SVS) on gradient-echo MRI are associated with a high percentage of red blood cells (mean of $48 \%$ ) and a low content of fibrin (mean of 26.4\%) and platelets (mean of 22.6\%) in clots. ${ }^{5,21,22}$ Moreover, a recent study showed that the susceptibility vessel sign can anticipate the macroscopic aspect of the retrieved clots with a good prediction accuracy: a SVS+ thrombus is likely not a 'white' 

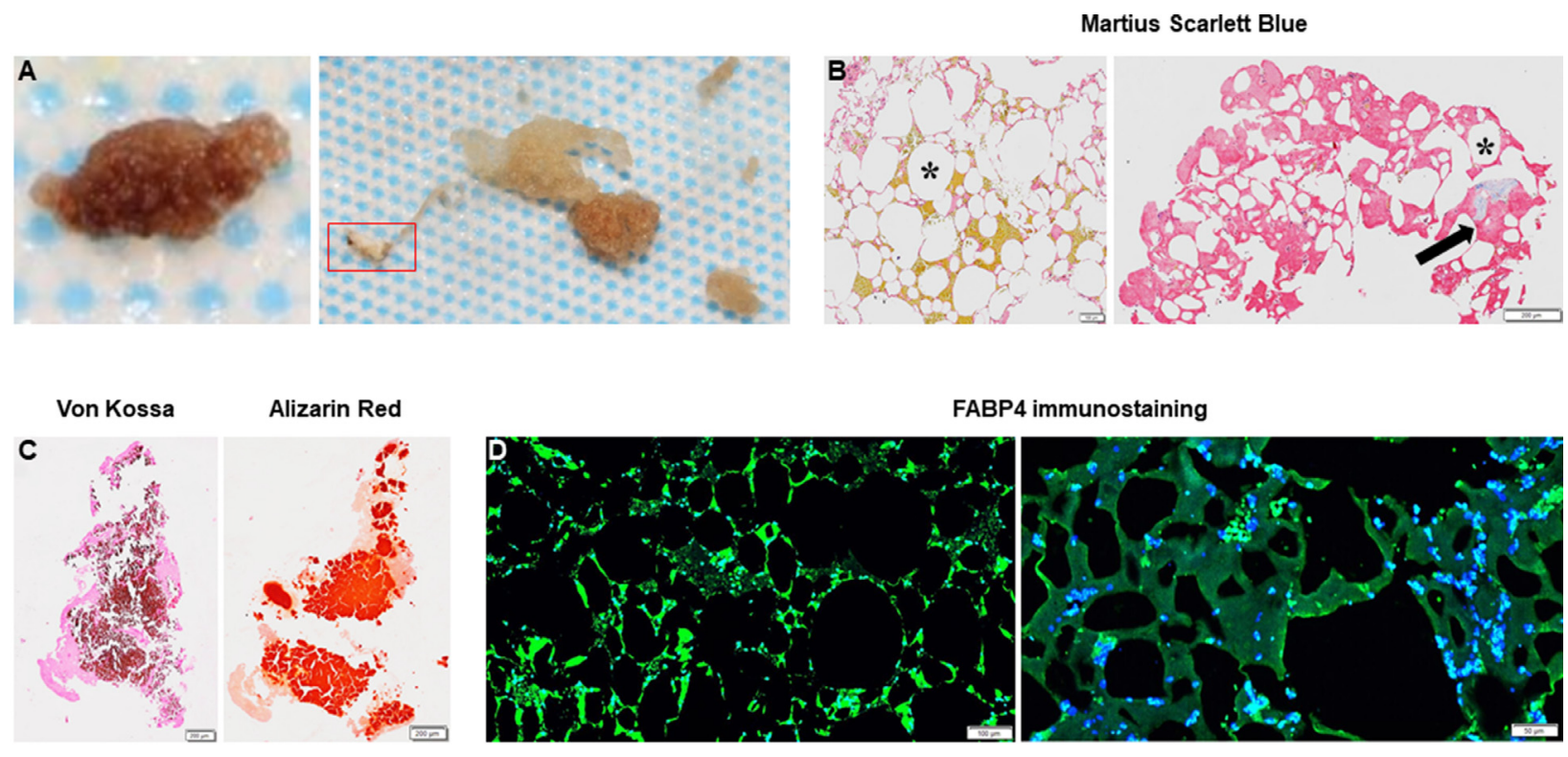

FABP4 immunostaining
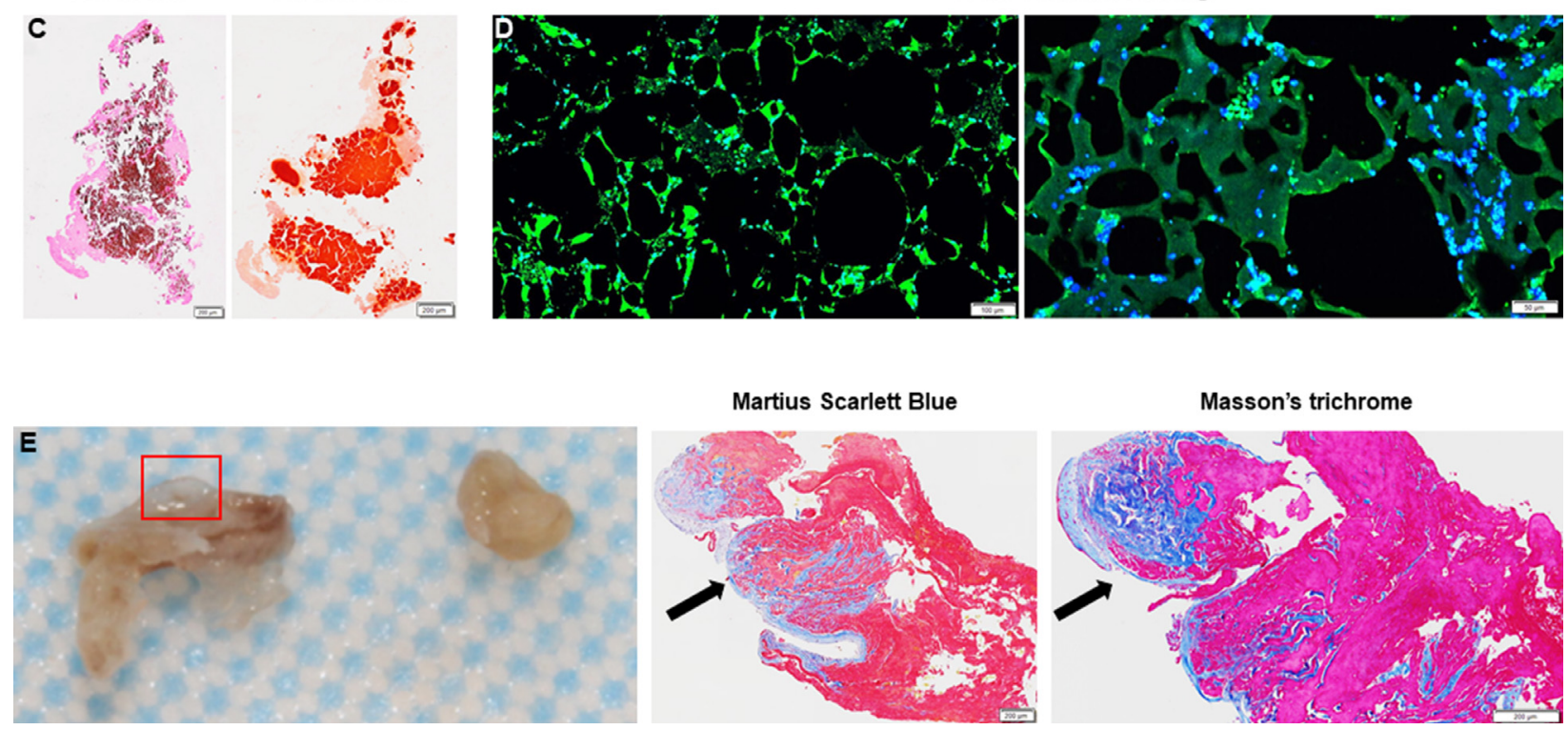

Fig. 3. Calcification, collagen and adipocytes are present in 'white' clots. Gross photos of two representative cases (A). MSB staining (B) showing adipocyte-like structures (star) and collagen (bright blue, arrow). Magnifications: 40x, 20x. Calcification suspected on gross examination ( $A$, red square), confirmed by von Kossa staining $(C$, brown). Alizarin Red staining demonstrating mineralization $(C$, orange-red). Magnification: 20x. FABP4 expressed by adipocytes in the first case and nuclei adjacent to adipocytes in the second case (D, green fluorescence). Nuclei stained with DAPI (blue fluorescence). Magnification: $100 x$. Presence of collagen ( $E$, red square) identified on MSB stain and confirmed by Masson's trichrome stain (blue, arrow). Magnifications: 30x, 40x.

thrombus. ${ }^{23}$ Therefore, if a 'white' clot is suspected to cause the occlusion, our data suggests a stentriever may be more effective to extract the clot.

Our study has limitations. Clots that were not removed by MT were not assessed in this study. Chemical and physical manipulation of clots may lead to mechanical-induced alteration, perhaps causing under or overrepresentation of some characteristics presented. Formalin fixation may change the macroscopic appearance and processing through xylene causes the loss of lipid content. The etiology and reperfusion outcome were self-reported at each site which may represent a source of variability. However, the relatively large number of 'white' clots in this cohort reduces the impact of some of these limitations.

\section{Conclusion}

'White' clots represented a distinct subset of clots extracted from AIS patients. They were characterized by platelet, vWF and collagen/calcification-rich composition consistent with a stiff thrombus material. 'White' clots were smaller and less hyperdense on NCCT than the other clots. They were associated with a high frequency of more distal occlusion locations and more often required switching approach to stentrievers during MT. Our findings may help to better understand the characteristics of difficult to remove clots such as 'white' clots.

\section{Funding}

This work has emanated from research conducted with the financial support of Science Foundation Ireland and European Regional Development Fund (Grant Number 13/RC/2073_2) and Cerenovus. It was also supported by the National Institutes of Health (R01 NS105853).

\section{Data availability}

Data are available from the corresponding author upon reasonable request. 


\section{Declaration of Competing Interest}

None.

\section{Acknowledgements}

The authors wish to acknowledge the invaluable contributions made by the Interventional/Nursing and Clinical coordination teams at each of the sites included in the RESTORE registry.

\section{References}

1. Boeckh-Behrens T, Schubert M, Förschler A, et al. The impact of histological clot composition in embolic stroke. Clin Neuroradiol 2016;26:189-197.

2. Choi MH, Park GH, Lee JS, et al. Erythrocyte fraction within retrieved thrombi contributes to thrombolytic response in acute ischemic stroke. Stroke 2018;49:652-659.

3. Maekawa K, Shibata M, Nakajima H, et al. Erythrocyterich thrombus is associated with reduced number of maneuvers and procedure time in patients with acute ischemic stroke undergoing mechanical thrombectomy. Cerebrovasc Dis Extra 2018;8:39-49.

4. Marder VJ, Chute DJ, Starkman S, et al. Analysis of thrombi retrieved from cerebral arteries of patients with acute ischemic stroke. Stroke 2006;37:2086-2093.

5. Niesten JM, van der Schaaf IC, van Dam L, et al. Histopathologic composition of cerebral thrombi of acute stroke patients is correlated with stroke subtype and thrombus attenuation. PLoS One 2014;9:e88882. https:/ / doi.org/10.1371/journal.pone.0088882.

6. Boodt N, Compagne KCJ, Dutra BG, et al. Stroke etiology and thrombus computed tomography characteristics in patients with acute ischemic stroke: a MR CLEAN Registry substudy. Stroke 2020;51:1727-1735.

7. De Meyer SF, Andersson T, Baxter B, et al. Analyses of thrombi in acute ischemic stroke: A consensus statement on current knowledge and future directions. Int J Stroke 2017;12:606-614.

8. Sgreccia A, Duchmann Z, Desilles JP, et al. Association between acute ischemic stroke etiology and macroscopic aspect of retrieved clots: is a clot's color a warning light for underlying pathologies? J Neurointerv Surg 2019;11:1197-1200.

9. Rossi R, Fitzgerald S, Gil SM, et al. Correlation between acute ischaemic stroke clot length before mechanical thrombectomy and extracted clot area: impact of thrombus size on number of passes for clot removal and final recanalization. Eur Stroke J Published Online First 2021. https: / / doi.org/10.1177/23969873211024777. 7 July.

10. Fitzgerald S, Rossi R, Mereuta OM, et al. Large artery atherosclerotic clots are larger than clots of other stroke etiologies and have poorer recanalization rates. J Stroke
Cerebrovasc Dis 2021;30:105463. https://doi.org/ 10.1016/j.jstrokecerebrovasdis.2020.105463.

11. Fitzgerald ST, Wang S, Dai D, et al. Platelet-rich clots as identified by Martius Scarlett Blue staining are isodense on NCCT. J Neurointerv Surg 2019;11:1145-1149.

12. Fitzgerald S, Wang S, Dai D, et al. Orbit image analysis machine learning software can be used for the histological quantification of acute ischemic stroke blood clots. PLoS One 2019;14:e0225841. https://doi.org/10.1371/ journal.pone.0225841.

13. Towler DA, Demer LL. Thematic series on the pathobiology of vascular calcification: an introduction. Circ Res 2011;108:1378-1380.

14. Fitzgerald S, Rossi R, Mereuta OM, et al. Per-pass analysis of acute ischemic stroke clots: impact of stroke etiology on extracted clot area and histological composition. J Neurointerv Surg 2020. https://doi.org/10.1136/neurintsurg-2020-016966. neurintsurg-2020-016966.

15. Douglas A, Fitzgerald S, Mereuta OM, et al. Platelet-rich emboli are associated with von Willebrand factor levels and have poorer revascularization outcomes. J Neurointerv SurgPublished Online First 2019. https://doi.org/ 10.1136/neurintsurg-2019-015410. 4 November.

16. Di Meglio L, Desilles JP, Ollivier V, et al. Acute ischemic stroke thrombi have an outer shell that impairs fibrinolysis. Neurology 2019;93:e1686-e1698. https://doi.org/ 10.1212/WNL.0000000000008395.

17. Staessens S, Denorme F, François O, et al. Structural analysis of ischemic stroke thrombi: histological indications for therapy resistance. Haematologica 2020;105:498-507.

18. Dobrocky T, Piechowiak E, Cianfoni A, et al. Thrombectomy of calcified emboli in stroke. Does histology of thrombi influence the effectiveness of thrombectomy? J Neurointerv Surg 2018;10:345-350.

19. Agardh HE, Folkersen L, Ekstrand J, et al. Expression of fatty acid-binding protein 4/aP2 is correlated with plaque instability in carotid atherosclerosis. J Intern Med 2011;269:200-210.

20. Fitzgerald S, Mereuta OM, Doyle KM, et al. Correlation of imaging and histopathology of thrombi in acute ischemic stroke with etiology and outcome. J Neurosurg Sci 2019;63:292-300.

21. Liebeskind DS, Sanossian N, Yong WH, et al. CT and MRI early vessel signs reflect clot composition in acute stroke. Stroke 2011;42:1237-1243.

22. Kim SK, Yoon W, Kim TS, et al. Histologic analysis of retrieved clots in acute ischemic stroke: correlation with stroke etiology and gradient-echo MRI. AJNR Am J Neuroradiol 2015;36:1756-1762.

23. Bourcier R, Duchmann Z, Sgreccia A, et al. Diagnostic performances of the susceptibility vessel sign on MRI for the prediction of macroscopic thrombi features in acute ischemic stroke. J Stroke Cerebrovasc Dis 2020;29:105245. https:// doi.org/10.1016/j.jstrokecerebrovasdis.2020.105245. 